\title{
Online hypnotic psychotherapy with distant patients
}

\begin{abstract}
The Coronavirus (COVID-19) pandemic forced many psychotherapists using hypnosis with their patients to venture into unexplored and undiscovered territory. They were challenged not only by technologies such as videoconferencing and the written and unwritten codes of online behavior, but also by the primary and well established rules of hypnotic and psychotherapeutic arts, which became challenging and confusing in the new digital environment. In the current paper, I will present the challenges of online hypnotic psychotherapy (H-psychotherapy) for professionals. Some practical recommendations for H-psychotherapy utilizing teleconference equipment will be offered. Additionally, specific techniques and strategies for the online implementation of hypnosis will be presented. Subjective and inter subjective factors that have to be addressed during online H- psychotherapy will be emphasized and discussed. All of the elements mentioned above are essential for working online with hypnosis while helping create and develop a safe, effective, and therapeutic space that can bypass the technological, physical, and psychological barriers.
\end{abstract}

Keywords: hypnotic psychotherapy, online hypnosis, tele-hypnosis, remote hypnosis, distant patient, intersubjective hypnosis
Volume 13 Issue 6 - 2020

\author{
Joseph Meyerson \\ Department of Psychology, Bar Ilan University, Israel
}

Correspondence: Joseph Meyerson, Department of Psychology, Bar Ilan University, 6 Meron s.t, Kfar Yona 4033537, Israel, Fax 15336042525, Tel +972544443239,

Email hypnoclinic10@gmail.com

Received: November 04, 2020 | Published: December 29, 2020

\section{Introduction}

Social distancing has been used by many countries as a useful measure to address the COVID-19 pandemic. However, it has prevented the world from moving along well-developed and familiar paths. H-psychotherapy clinics were no exception. Some of the hypnotists who already used Skype, Zoom, and other videoconferencing software were energized and felt victorious and confident. Nevertheless, most clinicians who routinely applied hypnosis during psychotherapy in face-to-face interactions in their clinics were somewhat confused and challenged. The online therapeutic arena became divided into three geographically and conceptually distant places: the therapist location, the patient location, and the digital interconnecting space.

Consequently, well-known principles of hypnotic art were challenged by technological, geographical, and psychological obstacles. The familiar psychotherapeutic props of empathic and therapeutic presence and intervention were shaken by online conferencing procedures and setting intricacies. The present paper will deal with the challenges of converting online H-psychotherapy with distant patients into a safe and effective psychotherapeutic space and process. In the first section of the paper, I will relate some objective factors of online hypnotic interactions. Those will include the design and utilization of the therapists' and patients' physical locations, computers, and peripheral equipment operation.

Additionally, the digital space of the internet and videoconferencing software will be considered. In the second part of the paper, more subjective matters will be discussed. The personal preferences of therapists and patients in the context of online H-psychotherapy will be addressed. Specific hypnotic strategies and techniques for handling hypnotized distant patients will be presented. Finally, the challenge to develop a safe, inter subjective healing space that can bypass the technological, physical, and psychological barriers during hypnotic psychotherapy will be addressed, especially by focusing on the therapist's ability to 'hold' patients when in silence and to 'contain' patients' abreactive expressions when online. ${ }^{1,2}$

\section{Modern remote hypnosis}

Practicing hypnosis with distant patients by phone (tele-hypnosis) was initiated approximately fifty years ago and was described as a possible and efficient practice. ${ }^{3-7}$ Two decades have passed since the first trials using videoconferencing hypnosis (video-hypnosis) were introduced ${ }^{8}$ since then, not much has been written about it. ${ }^{8,9}$

Overall, videoconferencing has been accepted and used in many therapeutic fields, including psychotherapy and even psychoanalysis. ${ }^{10-12}$ It has been designated as a useful instrument for treating a variety of mental health conditions while considering patients' and therapists' preferences and limitations. ${ }^{11,13,14}$ It was found useful with patients who are located at a distance from their therapists or have relocated, have accessibility issues, or are physically and/ or psychologically challenged. Therapeutic videoconferencing is helpful in emergency situations and for busy professionals. ${ }^{8,12}$ Lately, it has filled the geographical and social gap that opened between patients and their therapists following distancing policies during the COVID-19 pandemic..$^{15}$ It can also grant psychotherapy participants freedom from geographical or material stagnation (absence of fixed places of living and working). Against this background, the lack of scientific and clinical writing concerning online H-psychotherapy is even more prominent.

This disregard can't be explained only by a rigid clinging to traditional ways of conducting therapy, difficulty dealing with uncertainty, or technology anxiety. Hypnotists are usually out-ofthe-box innovators who are not easily intimidated by the unexpected and unknown. They aspire to improve their professional performance even in the face of being perceived as professionals that use marginal practices. ${ }^{16,17}$ The reason for the neglect of online H-psychotherapy is concealed in the fact that H-psychotherapy is a genuinely challenging task for both the therapist and the patient. In the next sections, the challenges mentioned above will be discussed, and some solutions will be presented. 
It should be noted that on-demand, fixed, online hypnotherapy is in coincidental use by internet frequenters and is not different from pre-recorded video/audio sessions that have been in use for decades by therapists. ${ }^{6,18}$ First-timers can use this kind of hypnotherapy marginally and experientially, and those experienced in hypnotherapy can use it prophylactically and, at times, as self-hypnosis exercise. ${ }^{19}$ The current paper will not include this kind of hypnotherapy and will focus on online interactive H-psychotherapy.

\section{The setting development during online H-psychotherapy}

Face to face H-psychotherapy has agreed upon and specific characteristics, rules, and roles that can be addressed as a setting. ${ }^{20}$ Usually, the therapist is in control of the setting or is at least responsible for defining and safeguarding it. By doing so, the hypnotist helps patients feel protected and to enter safely into an altered state of hypnosis, allowing effective personal psychotherapy. ${ }^{21-23}$ It should be noted that every psychotherapeutic setting, H-psychotherapy included, has concrete and subjective elements. ${ }^{24} \mathrm{I}$ will address these elements separately.

In the course of preparing this paper, I discussed the problems that arise during online H-psychotherapy with architects and industrial designers. I explained to them that, during the session, I have to develop a safe and therapeutic space to work hypnotically with patients by interconnecting three geographically and conceptually distant areas: the therapist's location, the patient's location, and the digital interconnecting medium. 'You are talking about the "The Venn Room," they proclaimed (The Venn Room - Spacepopular, 2019). ${ }^{25}$ It is a future-oriented concept that will merge distant places into one shared virtual reality that blends two or more physical spaces into one interconnected and newly created space. It replicates existing physical spaces and connects them in many unexpected ways. During online H-psychotherapy, I thought to myself that we actually co-create 'The Venn Clinic' anew with every patient and in every session.

\section{The concrete elements of online}

\section{H-psychotherapy}

Two physical places have to be considered in creating a therapeutic setting for online H-psychotherapy - the therapist's location and the patient's. The therapist's part of the clinic has to be designed such that what is seen on the screen projects the atmosphere the therapist wants to create. The illumination has to be sufficient, and the distance from the online camera should be variable; this can be achieved by a wheeled chair and a spacious room. During therapy hours, disturbances from people or domestic animals, if not therapeutically planned, should be minimized. The patient's side of 'The Venn Clinic' is molded and performed by the patient. The therapist's flexibility with patient creativity depends on the therapist's theoretical grounds and the ability to recruit the atmosphere created by the patient in favor of the therapeutic process. One of my supervisees told me that a patient of his phoned him at the beginning of a planned Zoom session and asked him to begin and carry on the session by phone for a short time until she arrived at her home computer. During this teletherapy talk, the therapist heard the sound of flushing water in the background, which seemed to be from a toilet flush. He felt somewhat humiliated but didn't know how to respond. During the supervision, we discussed the patients' behavior as being regressive and the therapeutic interaction as probably being directed by the patient's use of projective identification defenses. This perspective helped the supervisee develop a new therapeutic strategy that contributed significantly to the progress of the therapy.
It seems that beyond the practitioner's flexibility and creativity in utilizing the patient's contribution to the setting establishment, some setting rules should be retained. Asking the patient to start a session on time, guiding him/her to find a safe and private place without interference from household members, and using sufficient lighting that should be switched on before the evening darkness develops are ground rules that should be retained. Additionally, the patient must prepare a comfortable place, with some pillows and blankets, to sit or lie down in front of the camera during the session.

The online space, including peripheral equipment, is the interconnecting element of The Venn Clinic. Wi-Fi stability and bandwidth, online cameras, microphones and headsets, computers, screens, and software apps become an essential part of preparing and creating a therapeutic space in a multi-locational therapeutic environment. Decent quality peripheral equipment, as well as a computer (preferably a large-screen, fast desktop), a good quality camera, and quality headphones, enable the therapist to be more confident and prevent burnout when working with multiple patients. The location of one's camera is an essential element, as placing the camera at eye level creates an atmosphere of person-to-person communication as opposed to the atmosphere created by placing it above or below eye level - one-down or one-up positions. It is vital to master the operation of videoconferencing software. As I usually use the Zoom app (Video Conferencing, Web Conferencing, Webinars, Screen Sharing, n.d.), I won't expand on its operation, as there is useful guidance online.

Nevertheless, two specific elements of working with Zoom should be considered. Opening the Waiting Room a few minutes before the beginning of the session helps patients feel more confident. Additionally, the function that enables one to fix the large image of the partner for a conversation on the screen ('pin') should be used to prevent the speaker from talking to a self-screen image.

A fundamental and essential element of safe online H-psychotherapy is setting a protocol for online cutoff. It is included in preparing the patient for hypnosis. Aside from regular perpetrating procedures (Meyerson, 2020), patients should be guided in the following manner: 'Sometimes, cutting can be developed in online communication. So if you [the patient] are uncertain whether I [the therapist] am with you, you can call me [the therapist] by name. If, after calling twice, no answer is received, you will proceed with de-hypnotizing procedures by counting from five to one, opening your eyes, and reconnecting with me.' This concrete, agreed-upon safety measure helps patients feel more comfortable in their visits in a digital world and grants legitimacy to their uncertainties (transference of reality) concerning the development of a safe and appropriate therapeutic environment. The last comment links us naturally with the next part of the paper that deals with the subjective elements of online H-psychotherapy.

\section{The subjective elements of online H-psychotherapy}

Before the COVID-19 pandemic, most patients and a considerable number of therapists referred to online therapy with some reservations. ${ }^{26,27}$ Social distancing policies forced them to reconsider online therapy as it was almost the only opportunity left to arrange for therapy. In this context, the personal preferences and reservations that therapists and patients have about working with online hypnosis should be taken into account and treated in the proper context.

The hypnotists who feel comfortable or even prefer virtual H-psychotherapy usually have at least one of the subjective reasons presented below: 
a. They belong to a community of HSP or highly sensitive persons, ${ }^{28,29}$ who utilize online communication as a buffer for highly saturated emotional interactions with patients.

b. They do not feel comfortable with their bodies during face-toface sessions, and online interactions free them from being over involved with their physique.

c. They prefer geographical flexibility that offers them and their patients wider options not limited by geographical and physical borders.

d. They regard technology as a useful assistant and not as a dangerous competitor that threatens to replace them.

Therapists who dislike online H-psychotherapy are, in general, uncertain of their mastery of online hypnosis, especially with complicated patients and during emotionally charged therapeutic situations. They are confident when surrounded by the familiar interiors of their clinics, cherish in-person communications and interactions, and are unfamiliar with technological innovations.

Patients can also be divided into two groups: one recognizes the gains from online guided hypnotherapeutic interaction, and the other is deterred by it. Patients who feel more comfortable and safe during online H-psychotherapy include the following:

i. Some patients have difficulty controlling aggressive and sexual drives and behaviors; online physical disembodiment is helpful to them for drive regulation.

ii. Abused patients who may feel anxious in the physical presence of their therapists feel more relaxed when separated by distance and safely connecting with online therapists in their home environments.

iii. Some narcissistic and authority-challenged patients need to be in control of the therapeutic setting by governing the interior of their own spaces.

Ambivalence toward hypnosis-assisted psychotherapy as a whole or, in particular, toward the therapist figure, and indecision and fear in the context of therapy termination, can trigger the rejection of continuation during the passage to online therapy. Additionally, patients who avoid web-assisted therapeutic solutions despite their suffering and need for therapy have extreme separation anxiety. They don't see online H-psychotherapy as an adequate answer to their suffering. Some patients refuse to take part in online H-psychotherapy as they do not feel safe in the setting that does not have well-defined borders and are at risk of potential 'leaking' of personal information.

The therapist should be conscious of their preferences or restraints toward online H-psychotherapy, and sensitive toward the reasons their patients favor or reject it. Understanding patients' reservations emphatically and addressing them with appropriate explanations can help the suffering patients to continue therapy immediately or in the near future. Some of my supervisees reported that they felt insulted and threatened by a patient's refusal of online therapy, which put the continuation of the therapy into question.

\section{Specifics of online H-psychotherapy - techniques and strategies}

The belief that elicitation of hypnotic trance necessitates proximity and contact between the hypnotized and hypnotist is firmly rooted in the patients' cultural expectations and in some hypnotists' professional approach. ${ }^{30}$ These beliefs are based on a presupposition that the hypnotic process and influence have some concrete and material presence besides verbal and interpersonal interaction and communication. ${ }^{31-33}$ These beliefs are fueled by the history of hypnosis, onstage hypnosis performances, TV shows, and YouTube demonstrations of hypnosis and, in my view, contribute significantly to the withdrawal from online hetero-hypnosis. ${ }^{34,35}$

For effective online H-psychotherapy, the therapists' and patients' misconceptions have to be exposed and changed. After the misconceptions have been corrected, we have to bypass the difficulties that online therapy poses to us. These difficulties, among others, include two-dimensional on-screen vision; unreliable-at-times online communication; and distorted images, words, para-verbal intricacies, and body language, including 'minimal cues' that are so useful for hypnosis facilitation and handling. Thus, active and verbal patient participation is essential for developing and monitoring hypnotic trance. ${ }^{36}$ This can be achieved by teaching patients 'dissociative talk' ${ }^{37}$ and using question suggestions. ${ }^{23,38}$

At the beginning of the hypnotic session, after the induction, the following suggestion can be presented: 'And you can speak freely during the trance ... and with every word, you will go further into hypnosis ... Your speech will remain untouched, and at the same time, your body and your mind will continue their way deeper and deeper into hypnosis (dissociative talk). How do you feel right now? Can you now talk about ... ? Is it possible for you to concentrate on . . (question suggestions)?'

To complete the patients' adaptation to online hypnosis, the therapist should develop and use the 'therapist trance' extensively. This hypnotist stance can help to complete missing or fuzzy audio and video online information by more focused and refined attentive abilities facilitated by an externally focused, patient-oriented 'therapist trance.' After the patient's trance has sufficiently developed and the therapist has used the external patient-oriented 'hypnotist trance,' the next step in H-psychotherapy is synchronization between the patient and the therapist by developing a mutual co-trance, which sometimes can be called attunement. ${ }^{1}$

\section{The inter subjective elements of online H-psychotherapy}

H-psychotherapy is an interactive, intersubjective process, and this understanding has slowly but steadily penetrated into professional discourse..$^{2,39-42}$ Synchronized co-trance between the hypnotist and hypnotized has not only been theoretically discussed ${ }^{43,44}$ but has also been empirically researched..$^{43,45}$ During a face-to-face interaction, the trance of the patient meets and synchronizes with the trance of the hypnotist through neurocognitive mechanisms of 'mirror neurons ${ }^{42,46-48}$ mediated by vocal prosody, interpersonal synchrony, and nonverbal communication that takes place between hypnotist and hypnotized. ${ }^{2}$ Visual and auditory clues that are so important during face-to-face communication and serve as the basis of the therapistpatient attunement are weakened by the videoconferencing setting and online disturbances during online H-psychotherapy. To synchronize the patient's hypnotic trance with the therapist's trance, we have to rely on trance elements that are usually not located in the spotlight of clinical attention. The patient's externally oriented trance (p-EOT) and the therapist's internally oriented trance (t-IOT) can become leading instruments of attunement throughout H-psychotherapy with a distant patient. ${ }^{1}$ During hypnosis implementation, specifically after the induction, the therapist usually orients the patient's attention to the internal body-mind processes (p-IOT) and, at the same time, orients himself attentively toward the patient's verbal and physical 
behavior (t-EOT). Parallel to this process, the patient's externally focused attention (p-EOT) and the therapist's sensitivity to his interpersonal processes (t-IOT) are almost automatically developed and are extremely important during online H-psychotherapy. An externally oriented trance can help the patient be more attuned to the therapist, and the internally oriented trance of the therapist can be useful in allowing the therapist to use reminiscences of past hypnotic f2 $f$ interactions to complete the missing or Web-distorted elements of hypnotic interaction. Additionally, an internally oriented hypnotist trance can facilitate the hypnotist's clinical intuition by effectively using 'revere' experiences.

Appropriate coping with two very challenging hypnotherapeutic interactions, silence and intense emotional expression (abreaction), can enable the creation of a safe hypnotic and therapeutic space beyond the technological, physical, and psychological barriers. Usually, in f2f hypnotic interaction, silence is a unidirectional process: the patient remains silent, and the therapist talks or vice versa. The therapist's ability to be quiet during the patient's silence develops the patient's ability to be alone in the presence of others, ${ }^{49}$ thus increasing the patient's confidence and ability to work online Usually, during hypnotic psychotherapy, patients spontaneously take some time to be silent and process some important issues that have arisen during the hypnotic session..$^{50}$ It is the therapist's responsibility to recognize and allow this full-of-meaning silence without premature interruption. If the patient is using hollow verbalizations that mask the anxiety regarding staying silent, it is useful to initiate quietness and invite the patient 'to be quiet for a moment or two and to allow the issues addressed during the session to be submerged.' Additionally, the therapist's ability to be calm and empathic during online $\mathrm{H}$-psychotherapy as a patient forcefully expresses emotions signals to the patient that, although the therapist is distant, he/she can handle and contain the patient's emotional expressions.

\section{Reservations}

Online H-psychotherapy is a fruitful but somewhat complicated therapeutic modality, and as with every type of therapy, some safety measures have to be implemented. First of all, some guidance will be needed for all online newcomers, and supervision is essential for the therapist who feels incompetent in or uncertain about hypnosis application. Working with a patient who has been in f2f H-psychotherapy before is preferable. Patients with PTSD, personality disorders, or suicidal tendencies should be treated only by a specialist in these very sensitive mental health areas with appropriate precautions, such as communication with nearby, accessible, and trusted family members, friends, or neighbors.

\section{Conclusions}

Online H-psychotherapy, which has erupted into the consciousness of most therapists with the COVID-19 pandemic, will stay with us even after the epidemic passes. The opportunities that it reveals will gradually spread among the clinicians and their patients. Our ability, as professionals, to be flexible in the application of the therapy modes we promote and deliver to our patients will define our future relevancy as specialists in the mental health arena.

\section{Acknowledgments}

None.

\section{Conflicts of interest}

Author declares that there are no conflicts of interest.

\section{Funding}

None.

\section{References}

1. Baker EL. Reflections on the hypnotic relationship: Projective identification, containment, and attunement. The International Journal of Clinical and Experimental Hypnosis. 2000;48(1):56-69.

2. Spiegel EB, Baker EL, Daitch C, et al. Hypnosis and The Therapeutic Relationship: Relational Factors of Hypnosis in Psychotherapy. Am J Clin Hypn. 2020;62(1-2):118-137.

3. Aviv A. Tele-hypnosis in the treatment of adolescent school refusal. The American Journal of Clinical Hypnosis. 2006;49(1):31-40.

4. Kroger WS. Hypnotherapy for Intractable Post-Surgical Hiccups. American Journal of Clinical Hypnosis. 1969;12(1):1-4.

5. Owens HE. Hypnosis by Phone. American Journal of Clinical Hypnosis. 1970;(1):57-60.

6. Weitzenhoffer AM. Open-Ended Distance Hypnotherapy. American Journal of Clinical Hypnosis. 1972;14(4):236-248.

7. Wollman L. Clinical pathfinder no. 4: Hypnosis and the telephone. J Am Soc Psychosom Dent Med. 1978;25(4):126-128.

8. Simpson S, Morrow E, Jones M, et al. Video-Hypnosis - the Provision of Specialized Therapy via Videoconferencing. J Telemed Telecare. 2002;8 Suppl 2:78-79.

9. Hasan SS, Pearson JS, Morris J, et al. Skype Hypnotherapy for Irritable Bowel Syndrome: Effectiveness and Comparison with Face-to-Face Treatment. Int J Clin Exp Hypn. 2019;67(1):69-80.

10. Kocsis BJ, Yellowlees P. Tele-psychotherapy and the Therapeutic Relationship: Principles, Advantages, and Case Examples. Telemed J E Health. 2018;24(5):329-334.

11. Varker T, Brand RM, Ward J, et al. Efficacy of synchronous telepsychology interventions for people with anxiety, depression, posttraumatic stress disorder, and adjustment disorder: A rapid evidence assessment. Psychol Serv. 2019;16(4):621-635.

12. Weinberg H, Rolnick A. Theory and Practice of Online Therapy: Internet-delivered Interventions for Individuals, Groups, Families, and Organizations. $1^{\text {st }}$ edn. Routledge; 2019. p. 292.

13. Machado D de B, Laskoski PB, Severo CT, et al. A Psychodynamic Perspective on a Systematic Review of Online Psychotherapy for Adults. British Journal of Psychotherapy. 2016;32(1):79-108.

14. Scharff JS. Psychoanalysis Online: Mental Health, Teletherapy, and Training. USA: Routledge; 2013.

15. Crowe M, Inder M, Farmar R, et al. Delivering psychotherapy by video conference in the time of COVID-19: Some considerations. J Psychiatr Ment Health Nurs. 2020;1-2.

16. Meyerson J, Gelkopf M, Golan G, et al. What motivates professionals to learn and use hypnosis in clinical practice? Int J Clin Exp Hypn. 2013;61(1):71-80.

17. Strauss. Operator variables in Hypnotherapy. In Handbook of Clinical Hypnosis. 3rd edn. USA: American Psychological Association. 1997. pp. $55-72$

18. Hoslin L, Motamed C, Maurice-Szamburski A, et al. Impact of hypnosis on patient experience after venous access port implantation. Anaesth Crit Care Pain Med. 2019;38(6):609-613.

19. Farrell-Carnahan L, Ritterband LM, Bailey ET, et al. Feasibility and preliminary efficacy of a self-hypnosis intervention available on the Web for cancer survivors with insomnia. E-Journal of Applied Psychology. 2010;6(2):10-23. 
20. Mayer A. Lost Objects: From the Laboratories of Hypnosis to the Psychoanalytic Setting. Sci Context. 2006;19(1):37-64.

21. Erickson MH, Rossi EL. Hypnotherapy: An Exploratory Casebook (Har/ Cas). USA: Ardent Media; 1979. p. 351.

22. Gilligan S. Therapeutic Trances: The Co-Operation Principle in Ericksonian Hypnotherapy. 1st edn. Routledge. 1986. p. 386.

23. Yapko MD, Sheehan P. Trance work: An Introduction to the Practice of Clinical Hypnosis. $4^{\text {th }}$ edn. USA: Routledge; 2012

24. Lunn S. The psychoanalytic room. Scand Psychoanal Rev. 2002;135-142.

25. http://www.spacepopular.com/exhibitions/2019—-the-venn-room

26. Paslakis G, Fischer-Jacobs J, Pape L, et al. Assessment of Use and Preferences Regarding Internet-Based Health Care Delivery: CrossSectional Questionnaire Study. J Med Internet Res. 2019;21(5):e12416.

27. Renn BN, Hoeft TJ, Lee HS, et al. Preference for in-person psychotherapy versus digital psychotherapy options for depression: Survey of adults in the U.S. NPJ Digit Med. 2019;2:6.

28. Aron A, Ketay S, Hedden T, et al. Temperament trait of sensory processing sensitivity moderates cultural differences in neural response. Soc Cogn Affect Neurosci. 2010;5(2-3):219-226.

29. Aron EN. The Highly Sensitive Person. USA: Kensington Publishing Corp; 2013. p. 288

30. Levitan AA, Johnson JM. The Role of Touch in Healing and Hypnotherapy. Am J Clin Hypn. 1986;28(4):218-223.

31. Capafons A, Mendoza ME, Espejo B, et al. Attitudes and beliefs about hypnosis: A multicultural study. Contemporary Hypnosis. 2008;25(34):141-155.

32. Green JP. Beliefs about Hypnosis: Popular Beliefs, Misconceptions, and the Importance of Experience. International Journal of Clinical and Experimental Hypnosis. 2013;51(4):369-381.

33. Meyerson J. The myth of hypnosis: The need for remythification. Int $J$ Clin Exp Hypn. 2014;62(3):378-393.

34. Pintar J, Lynn SJ. Hypnosis: A brief history. USA: Wiley-Blackwell; 2008. p. 240

35. Rosenfeld SM. A critical history of hypnotism: The unauthorized story. Bloomington, Indiana: Xlibris; 2008.

36. Meyerson J. Self-Talk Monitoring and Utilization for Enhancing Hypnotic Induction. Am J Clin Hypn. 2017;60(2):149-158.

37. Milton H. Erickson. Teaching Seminar with Milton H. Erickson. $1^{\text {st }}$ edn. Routledge, USA: Psychology Press; 1985.
38. Battino TLSR. Ericksonian Approaches: A Comprehensive Manual. 2nd edn. UK: Crown House Publishing; 2005.

39. Alter DS. In the Intersubjective Space: Hypnosis through a Neuropsychological Lens. Am J Clin Hypn. 2020;62(1-2):74-94.

40. Bonshtein U. Relational hypnosis. Int J Clin Exp Hypn. 2012;60(4):397415.

41. Diamond MJ. The long and winding road from concept to practice: The intersubjective shaping of psychoanalytically informed technique in contemporary hypnosis - a commentary upon and extension of Baker's "reflections on the hypnotic relationship. Int J Clin Exp Hypn. 2000;48(1):70-85.

42. Henning JA. An Inter subjective View of Empathy and Hypnotic Trance: Response to Wickramasekera II. The American Journal of Clinical Hypnosis. 2016;58(3):256-273.

43. Varga K. The phenomenology of hypnotic interactions: towards a new synchron phenomenon. Magyar Pszichol\&oacute; Giai Szemle. 2019;74(1):97-114.

44. Varga K, Józsa E, Bányai ÉI, et al. A new way of characterizing hypnotic interactions: Dyadic Interactional Harmony (DIH) questionnaire. Contemporary Hypnosis. 2006;23(4):151-166.

45. Varga K, Kekecs Z. Oxytocin and Cortisol in the Hypnotic Interaction1. International Journal of Clinical and Experimental Hypnosis. 2014;62(1):111-128.

46. Bråten S. On Being Moved: From Mirror Neurons to Empathy. USA: John Benjamins Publishing; 2007.

47. Gallese V, Eagle MN, Migone P. Intentional Attunement: Mirror Neurons and the Neural Underpinnings of Interpersonal Relations. J Am Psychoanal Assoc. Winter. 2007;55(1):131-176.

48. Rossi E L, Rossi KL. The Neuroscience of Observing Consciousness \& Mirror Neurons in Therapeutic Hypnosis. Am J Clin Hypn. 2006;48(4):263-278.

49. Winnicott DW. The Capacity to be Alone. Int J Psycho-Anal. 1958;39:416-420.

50. Rossi E, Mortimer J, Rossi K. Therapeutic Hypnosis, Psychotherapy, and the Digital Humanities: The Narratives and Culturomics of Hypnosis, 1800-2008. American Journal of Clinical Hypnosis. 2013;55(4):343-359.

51. Meyerson J. Presenting hypnosis to patients. In Elkins GR editor. Handbook of medical and psychological hypnosis. New York, USA: Springer; 2017; p. 29-34

52. Lunn S. The psychoanalytic room. Scand Psychoanal Rev. 2002;135-142.

53. https://zoom.us/ 